\title{
Nonrandom partition of mitochondria in heteroplasmic Drosophila
}

\author{
ERIC DE STORDEUR \\ Laboratoire de Zoogéographie, Université Paul Valéry, Route de Mende, F 34199 Montpellier CEDEX 5, France
}

\begin{abstract}
In order to understand the status of heteroplasmy and its evolution within the Drosophila melanogaster subgroup, cytoplasm microinjections between eggs were performed involving three lineages of Drosophila simulans, carrying the siI, siII or siIII mtDNA type, respectively, and two strains of Drosophila mauritiana carrying the maI or maII mtDNA type. Progeny of eggs from all combinations of injection were analysed. The maII or siI molecules, when provided by the donor, were never detected in the offspring of the hosts, whatever the host's mitochondrial type. Heteroplasmic flies were detected when siII, siIII or maI mitochondria were injected into any of the other cytoplasms. In the majority of cases the percentage of foreign mtDNA increased over generations, leading to a complete replacement of the endogenous mtDNA. In most cases, siII was prevalent. The stochastic processes involved in the evolution of heteroplasmic states are strongly affected by selective values of the different mtDNA types, with a clear hierarchy among them: siII has the most advantage, then siIII and $m a \mathrm{I}$, and finally siI and $m a \mathrm{II}$. In the siII/maII heteroplasmy case, the loss of $m a \mathrm{II}$ was more rapid at a high temperature.
\end{abstract}

Keywords: Drosophila, heteroplasmy, mitochondria, mtDNA, nuclear-cytoplasmic interactions, selection.

\section{Introduction}

Animal cells harbour several thousands of mitochondrial DNA (mtDNA) molecules distributed among several hundreds of mitochondria. In spite of this high level of physical polyploidy, they are most often genetically haploid, i.e. all their mtDNA molecules are identical. However, an increasing number of individuals carrying more than one type of mtDNA (usually described as heteroplasmic) is being detected in the animal kingdom. Their appearance can be related to two mechanisms according to mtDNA genetics.

1 Maternal inheritance of mtDNA is the rule for most animal species studied so far; it was demonstrated many years ago (Dawid \& Blackler, 1972) and can be inferred when crosses between individuals (from the same species or related ones) that harbour different mtDNA types produce progeny that exhibit only the maternal mtDNA. Given that situation, the origin of zygote heteroplasmy is limited to mutations occurring in female germ cells and the new mtDNA molecules are expected to

\footnotetext{
*Correspondence. E-mail: stordeur@bred.univ-montp3.fr
}

differ by a single mutation step (base substitution, addition/deletion of a repeated sequence, large deletion). Resolution of such a heteroplasmy has been analysed in few cases: Drosophila (Solignac et al., 1983, 1984; Volz-Lingenhohl et al., 1992), crickets (Rand \& Harrison, 1986), cows (Hauswirth \& Laipis, 1985) and rabbits (Casane et al., 1994). The time required for mtDNA segregation/purification depends on several variables. Two basic ones are the number of segregating units at each cell division (approximately the number of mitochondria) and the mutation rate; some variables are incidental: mode of cell division in the germ line and selection for or against the mutant molecule. This time varies from several hundred generations for Drosophila and crickets to only one for cows; individuals with more than two mtDNA types can be observed whenever mutated molecules arise faster than they are purified. But in some cases heteroplasmy is never resolved, e.g. Drosophila subobscura (Volz-Lingenhohl et al., 1992) and rabbits (Casane et al., 1994), and generalized heteroplasmy has even been described when every individual is heteroplasmic, harbouring a population of length-variable molecules, e.g. frogs (Monnerot et al., 1984) and rabbits 
(Ennafaa et al., 1987). Selective pressures have been identified (Solignac et al., 1987; Casane et al., 1994) and they may vary according to cell differentiation (Casane et al., 1994).

2 Paternal contribution can be suspected whenever heteroplasmic individuals carry very divergent mtDNA molecules. It was first demonstrated in two cases of experimental interspecific crosses of mice (Gyllensten et al., 1991) and Drosophila (Satta et al., 1988; Kondo et al., 1990). Since then significant paternal contributions have been inferred from observations of mussel (Hoeh et al., 1991) and anchovy (Magoulas \& Zouros, 1993) populations. Crosses between Mytilus edulis with rather divergent mtDNA showed total exclusion of paternal mtDNA within oocytes, whereas incomplete exclusion of maternal mtDNA took place within sperm (Zouros et al., 1992; Skibinski et al., 1994). Following a paternal contribution, a given individual has heteroplasmic somatic cells whenever its parents did not carry the same mtDNA type and the nature of its progeny (homoplasmic or heteroplasmic) will mostly depend on the type of mtDNA of its mate.

The main variables involved in the genetics and evolution of the mitochondrial genome are thus: (i) the relative contribution of the two parents to the genotype of the offspring - uniparental caused by the exclusion, destruction or dilution of one genome, or biparental, occasionally sex-dependent or associated with interspecific crosses; (ii) partitioning at cell division; (iii) selective values of the various genotypes.

Because spontaneous heteroplasmy is not very frequent in metazoans, experimental heteroplasmic individuals were necessary for further investigations on the relationships between nucleus and mitochondria and between different types of mitochondria. We have already obtained heteroplasmic Drosophila simulans through cytoplasm additions in eggs carrying siII or siIII mitochondrial DNA (de Stordeur $e t$ al., 1989): the distribution of the mitochondrial genotypes in the offspring of heteroplasmic females suggested that (i) the stochastic processes involved in the resolution of this multiple nucleotide sites heteroplasmy were very similar to those previously described for spontaneous length heteroplasmy (Solignac et al., 1984), and (ii) the siII mitochondrial genome was prevalent over the siIII one. Experimental heteroplasmic $D$. melanogaster were also produced by Matsuura et al. (1989) and Niki et al. (1989) through injection of $D$. mauritiana germ plasm; they reported the complete replacement of resident mtDNA in some lines. Matsuura et al. (1991, 1993) observed the selective transmission of one or the other mtDNA depending on the temperature; they suggested that the two mtDNAs could compete in replication efficiency.

The present paper reports the evolution of mtDNA frequencies in the progeny of heteroplasmic $D$. simulans and D. mauritiana obtained through intraspecific and interspecific microinjections of cytoplasm, and documents the occurrence of selective processes. In one case the changes in two mtDNA frequencies were examined at different temperatures.

\section{Materials and methods}

\section{Drosophila strains}

The experiments involved the two closely related species Drosophila simulans and D. mauritiana, the mtDNA of which has already been described (Solignac et al., 1986). For D. simulans three isofemale strains carry the following mtDNA types: siI (Seychelles line), siII (Villeurbanne line) and siIII (Mont d'Ambre line), respectively. The siI mtDNA type is known in Indo-Pacific regions, the siII type is present worldwide and siIII only in Madagascar and La Réunion. The two $D$. mauritiana strains also differ by their mtDNA type: $m a I$ (G20.4 line) or maII mtDNA (G52 line) (Solignac et al., 1986). Drosophila mauritiana is endemic to Mauritius.

Comparisons of restriction maps showed that the differences between the mitochondrial genomes taken two-by-two ranged from 1.5 to 3.3 per cent (Solignac et al., 1986), except for siIII and maI which are indistinguishable with restriction enzymes; this mtDNA type is named according to the species which carries it.

Wolbachia, a rickettsia-like bacterium which is a symbiont of Drosophila, has been detected only in the siII strain. This bacterium is known to be responsible for cytoplasmic incompatibility (Binnington \& Hoffmann, 1989). No problem of fecundity was noticed in these experiments.

\section{Cytoplasm injection and experimental conditions}

The transfer of mitochondria was carried out by egg-to-egg microinjection of cytoplasm, using the technique described by Santamaria (1987). Donor and recipient eggs were at stage 2 (prior to pole cells formation). The chorion was removed for both, then the eggs were slightly dehydrated and finally 
the donor cytoplasm was injected into the posterior end of the host eggs.

Because of the identity of siIII and maI mtDNA types, only 18 types of injections among the possible 20 were carried out. The females obtained from the injected eggs were systematically mated to males from the host line in order to be sure that observed heteroplasmy was not caused by paternal leakage, if any. For each of these females 10-30 $F_{1}$ females were isolated to found as many sublines. Occasionally females were taken from further generations to create as many sublines. They will also be referred to as 'founder females'. When not specified, each generation is derived from at least 30 individuals belonging to the previous generation.

The strains and lines were reared at $21^{\circ} \mathrm{C}$. When the effect of temperature was studied, single heteroplasmic flies were allowed to lay eggs successively at $25^{\circ}, 17^{\circ}$ and $21^{\circ} \mathrm{C}$. The progeny were then maintained at the specified temperature.

Artificially created heteroplasmic individuals are identified by the name of the mtDNA type of the receptor cell followed by that of the donor: in an siIII/maII heteroplasmy, cytoplasm of an maII cell is injected into an sillI cell.

\section{DNA extraction and analysis}

Mitochondrial DNA heterogeneity in a line can result from either a mixture of homoplasmic individuals, the presence of heteroplasmic individuals, or a combination of both. An examination of the flies one-by-one is necessary to indicate that a line contains heteroplasmic flies.

mtDNA was extracted following the technique described by Latorre et al. (1986). Each DNA preparation was digested with enzymes generating diagnostic mtDNA fragments (even if samples are partially digested). These enzymes are listed in Table 1 . The restriction fragments were separated by electrophoresis on 1 per cent vertical agarose gels using a Tris/acetate/EDTA buffer. Because the DNA extracted is not pure mtDNA, it was trans-

Table 1 Enzymes used to distinguish the mtDNA types of Drosophila mauritiana and D. simulans

\begin{tabular}{lccc}
\hline & siII & siIII and $m a \mathrm{I}$ & maII \\
\hline si & $H p a \mathrm{I}$ & ClaI & HindIII \\
siII & - & $H p a \mathrm{II}$ or HpaI & HpaII \\
siIII and $m a \mathrm{I}$ & - & - & HpaII \\
\hline
\end{tabular}

ferred by Southern blotting to a nylon membrane (Pall Biodyne), hybridized to ${ }^{32} \mathrm{P}$-labelled purified $D$. simulans mtDNA and autoradiographed. The proportions of foreign and host mtDNAs were estimated (without correction for the length differences of the fragments) by scanning the appropriate bands on autoradiographs.

Usually, mtDNA type frequencies were studied in founder females and/or in their $F_{1}$ and in further generations (number of analysed individuals: 10-45). When temperature was an experimental variable, the founder female and its $F_{1}, F_{2}$ and $F_{7}$ were analysed. Frequencies of mtDNA types within a population at a given generation were estimated from the analysis of individuals taken one-by-one, and expressed as the mean of the frequencies observed for each of them, or, alternatively, from a pool of at least 10 individuals; this is indicated for each case. These two different approaches gave the same results even though the first one is more informative.

\section{Selective value}

The relative fitness per fly generation, $w$, was estimated when possible according to Solignac et al. (1984).

\section{Results}

Reciprocal injections involving five mitochondrial types were undertaken to examine nuclear-mitochondrial and intermitochondrial relationships. When the experiments are considered altogether, 180 female flies emerged from injected eggs; among them 33 flies provided heterogeneous progeny. These females, which can be considered as transplasmic, emerged from eggs which received and conserved alien cytoplasm in some pole cells. Table 2 gives, for each set of injections, the number of transplasmic females out of those that emerged. The $\chi^{2}$-test for injection success/failure according to the donor strains demonstrates that these results are not random $\left(\chi_{4}^{2}=94, P<0.001\right)$.

Depending on the level of the generation studied first in the mother of the $F_{1}$ progeny, the abscissae in Figs 1-3 start either at generation 0 or at generation 1.

\section{sil and mall as donor}

The siI and malI cytoplasms, when injected, were never detected in the offspring of the four possible 
Table 2 Number of Drosophila females that generated heteroplasmic offspring as a proportion of the number of females that emerged from injected eggs

\begin{tabular}{lccccc}
\hline Donor & \multicolumn{3}{c}{ Drosophila simulans } & \multicolumn{2}{c}{ Drosophila mauritiana } \\
\cline { 2 - 5 } Host & siI & siII & siIII & maI & maII \\
\hline siI & - & $3 / 13$ & $5 / 7$ & $1 / 7$ & $0 / 15$ \\
siII & $0 / 12$ & - & $3 / 14$ & - & $-1 / 7$ \\
siIII & $0 / 14$ & $10 / 25$ & - & - & $0 / 7$ \\
maI & $0 / 10$ & $2 / 6$ & $5 / 11$ & $2 / 7$ & $0 / 7$ \\
maII & $0 / 7$ & $1 / 1$ & &
\end{tabular}

hosts (43 and 39 emerged females, respectively), whatever their own mtDNA type.

\section{sill as donor}

When the siII cytoplasm was injected into siI eggs, three females among 13 provided heteroplasmic flies.
Thirty-eight isofemale lines (12 or 13 per female) were founded. Eleven heterogeneous lines were studied (pooled analysis) over two to 12 generations: the frequency of siII increased in all of them but one (Fig. 1a). The relative fitness of siII in competition with siI, $w_{s i I I}$, can thus be considered as higher than 1. (a)

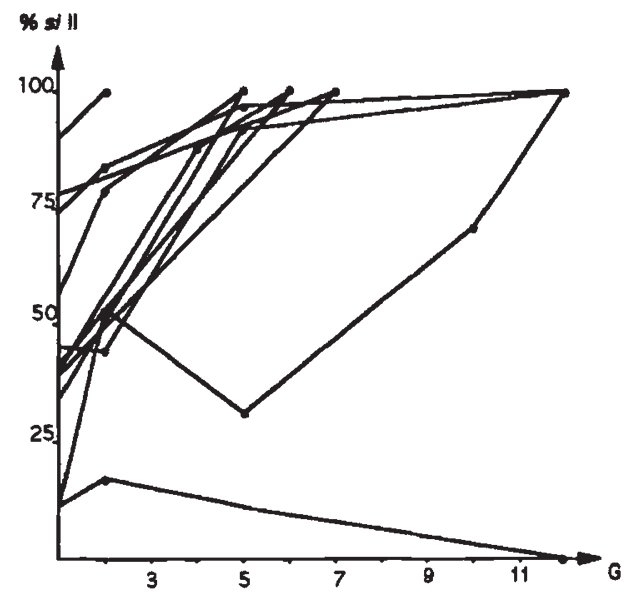

(c)

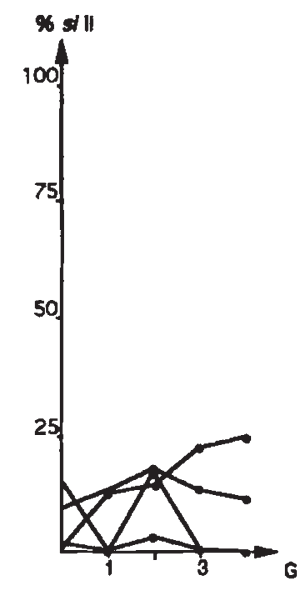

(b)

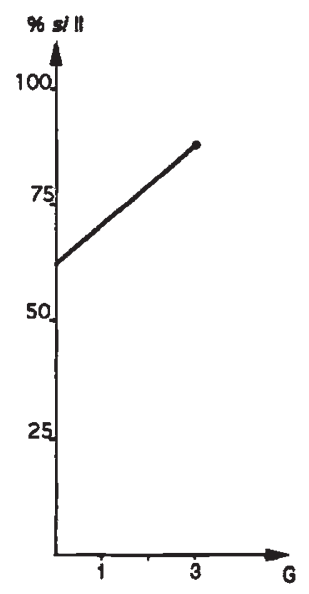

(d)

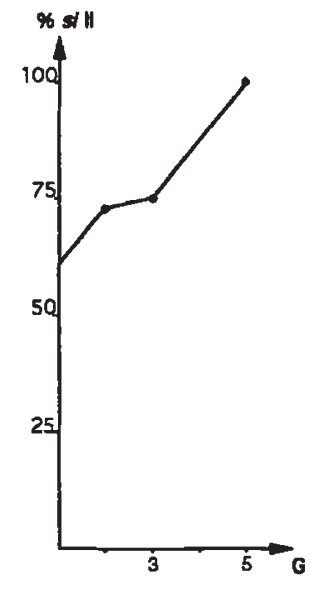

Fig. 1 Changes in foreign mtDNA frequencies in the progeny of heteroplasmic female Drosophila. G, generations. (a) Progeny of 11 si $/ /$ si II females; (b) progeny of one siIII/siII female; (c) progeny of four $\mathrm{maI} / \mathrm{si} \mathrm{II}$ females; (d) progeny of one maII/siII female. 

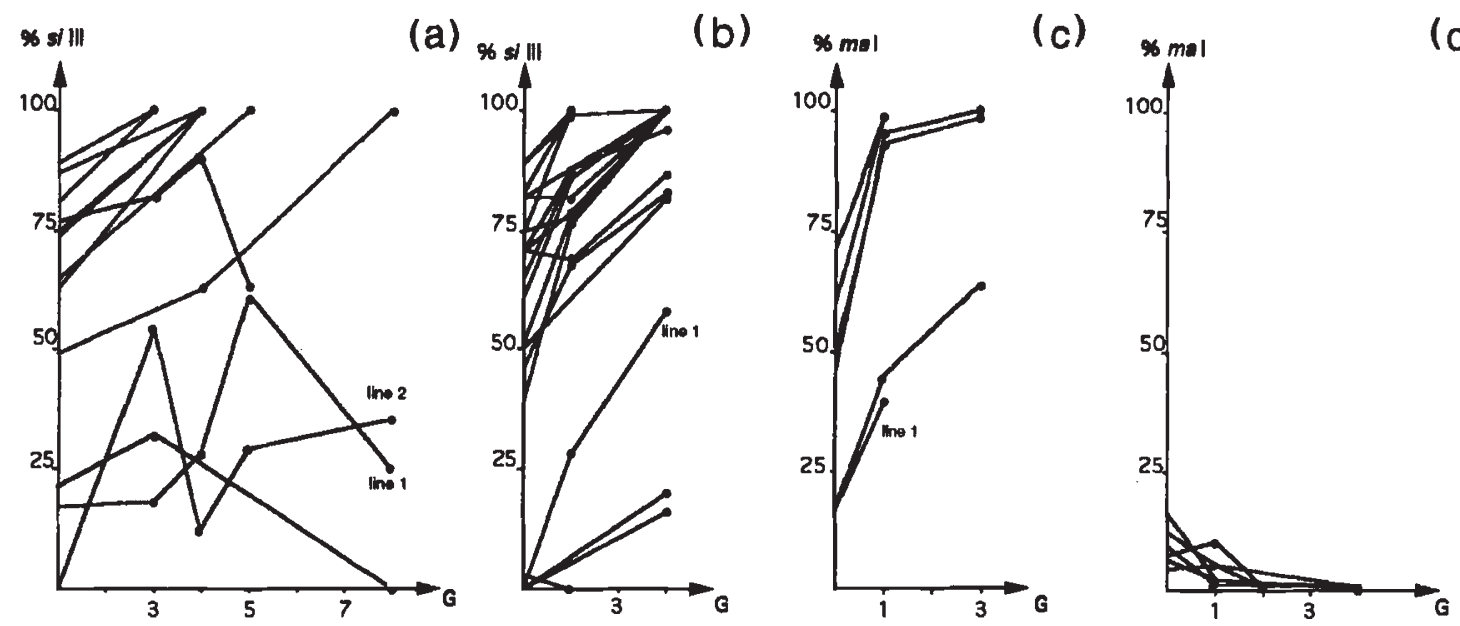

(d)

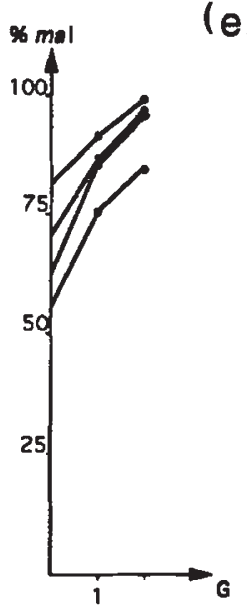

Fig. 2 Changes in the foreign mtDNA frequencies in the progeny of heteroplasmic female Drosophila. G, generations. (a) Progeny of 12 sil/silI females; (b) progeny of $20 \mathrm{maII} / \mathrm{siII}$ females; (c) progeny of six sil $/ \mathrm{maI}$ females; (d) progeny of six sill $/ m a I$ females; (e) progeny of four $m a I I / m a I$ females.

The situation where siII cytoplasm is injected into siIII eggs has been analysed previously (de Stordeur et al., 1989): most of the lines lost the siIII mtDNA. The frequencies of siIII and siII were determined in a single fly taken at the second generation, and in 44 individuals from its $\mathrm{F}_{3}$ progeny: the frequency of siII was 61.5 per cent in the female and 87.3 per cent in the $\mathrm{F}_{3}$ (Fig. 1b, 44 flies analysed individually). In this case, the relative fitness $w_{\text {sil }}$ is 1.58 .

Two heteroplasmic flies emerged from maI hosts; four lines among the 23 established from these two flies were studied individual-by-individual (10-30 flies). The siII mtDNA type was still present in two lines at generation $\mathrm{F}_{4}$; its frequency was low but increased slowly in one line (Fig. 1c).

Only one interspecific heteroplasmic siII/maII female emerged when maII was the host. Nine lines were created: only one among them was heterogeneous. The frequency of the siII mtDNA increased in this progeny over generations and the maII mtDNA was no longer detected (pooled analysis) at the fifth generation (Fig. 1d). The selective value, $w_{\text {sill }}$, calculated between $\mathbf{F}_{1}$ and $\mathrm{F}_{2}$ is 1.66.

\section{silll as donor}

When the host carried si m mDNA, five females among seven provided heterogeneous progeny and each initiated eight to 10 lines. Among the 45 lines thus founded, 12 were heterogeneous and were followed during five to eight generations. The siIII mtDNA frequency increased over generations in nine of them (pooled analysis) and the resident mtDNA was lost after three to eight generations (Fig. 2a). The relative fitness of silII in competition with siI, $w_{\text {sinl }}$, is thus higher than 1 . At the eighth generation, individual analysis of 24 and 23 flies from lines 1 and 2, respectively, still heterogeneous, demonstrated that every individual was homoplasmic, exhibiting either the siI or the siIII type.

Results of injecting siIII into siII eggs have been previously reported (de Stordeur et al., 1989): after 10 generations the foreign mtDNA was systematically lost in the seven lines analysed: $w_{s i l}$ is higher than 1.

Five interspecific siIII/maII females emerged and 10 lines per female were initiated. Among the 50 lines, 22 showed heterogeneity. The siIII frequency increased rapidly over generations in all of them except one. Thus $w_{\text {sillI }}$, in competition with maII, is higher than 1. The frequencies of mtDNAs were estimated from groups of about 10 individuals except for line 1 ( $23 \mathrm{~F}_{4}$ individuals analysed separately) (Fig. 2b). The experiments testing the effect of temperature started with $\mathrm{F}_{4}$ female flies from this protocol (see below).

\section{$\mathrm{ma}$ as donor}

Only one female was generated by injection of $m a \mathrm{I}$ into siI eggs. Line 1 was initiated from a heteroplasmic $F_{1}$ female. Lines $2-6$ were each initiated with one heteroplasmic $F_{1}$ female from line 1. The maI mtDNA confronted with the siI type increased 
very rapidly in the six lines (Fig. $2 \mathrm{c}$ ). The relative fitness, $w_{m a}$, is thus higher than 1. Each line was studied by individual analysis (10-31 flies).

The injection of maI into silI eggs provided one female; six lines were initiated from it. These lines were analysed individual-by-individual. The initial frequency of maI was low to begin with (4-16 per cent) and it dropped down to $0-0.5$ per cent after two to four generations (Fig. 2d): $w_{\text {sil }}>1$.

Two $m a \mathrm{I} / \mathrm{maII}$ females were obtained. Four lines were initiated from four sisters and the foreign mtDNA type mal increased rapidly in all lines, which were followed until the $\mathrm{F}_{2}$ (Fig. 2e, 11-28 flies analysed individually). The mean relative fitness of maI in competition with maII, calculated between the female and its $F_{1}$ offspring, is $w_{\text {maI }}=2.75 \pm 0.55$ (SE).

\section{Temperature effect}

The effect of temperature on the fate of heteroplasmy was tested on the $F_{1}, F_{2}$ and $F_{7}$ progeny of seven heteroplasmic siIII/maII flies analysed individually: the percentage of siIII mtDNA in these flies

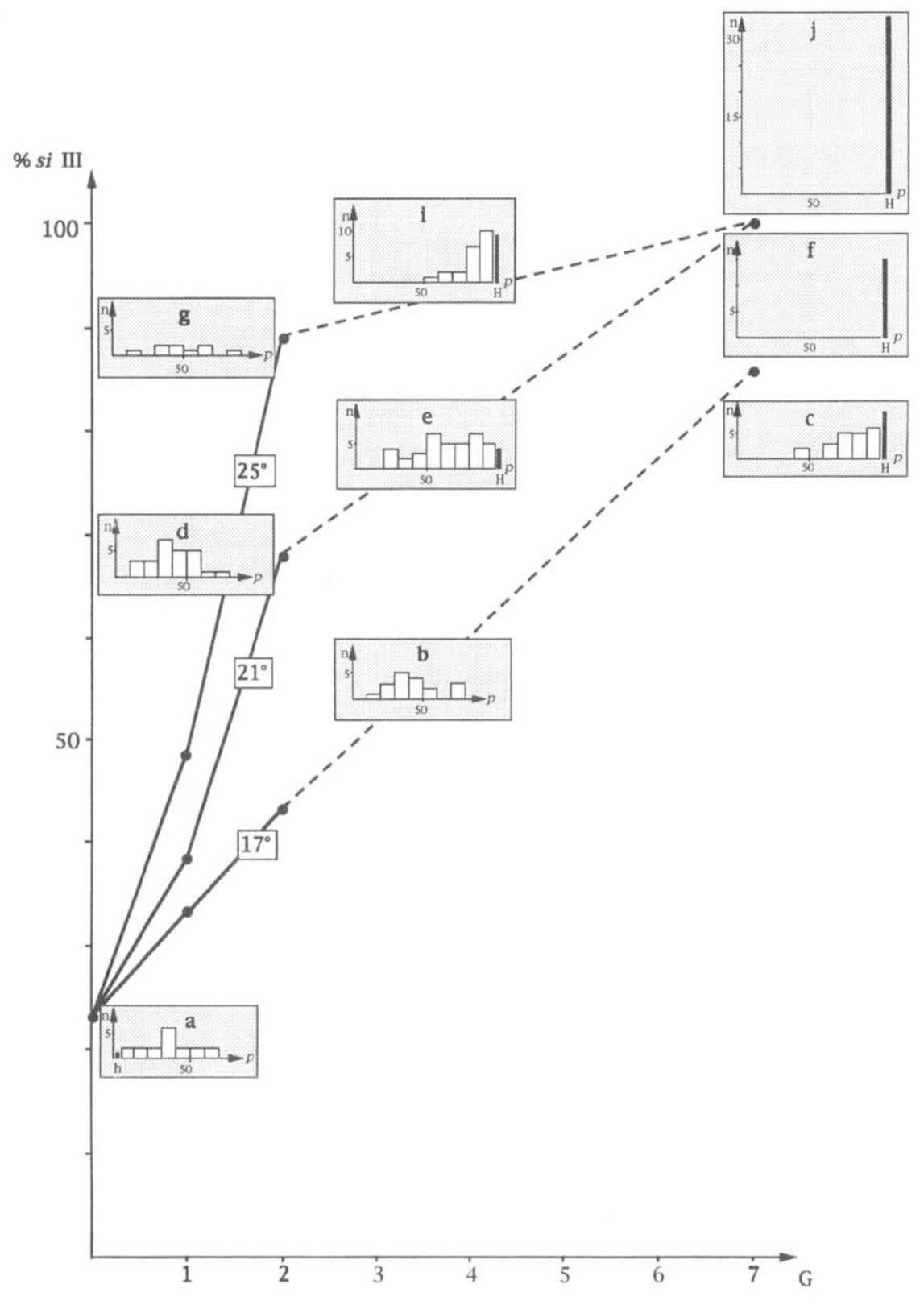

Fig. 3 Changes in the frequency of the siIII mtDNA type according to the temperature and the generation. The $F_{1}, F_{2}$ and $F_{7}$ offspring of one interspecific heteroplasmic $m a \mathrm{II} / s i \mathrm{III}$ Drosophila mauritiana female were reared at $17^{\circ}$ (histograms $\mathrm{a}, \mathrm{b}$ and $\mathrm{c}$ ), $21^{\circ}$ (histograms d, e and f) or $25^{\circ} \mathrm{C}$ (histograms g, i and j). G, generations. The lines between $F_{2}$ and $F_{7}$ are dotted because intermediate data are not available. Histograms: distribution of the percentages (class interval, 10 per cent) of the foreign mtDNA $(p)$ in the progeny of a single heteroplasmic fly; $n$, number of flies analysed; open rectangles, heteroplasmic individuals; black rectangles, individuals with $p>98$ per cent $(\mathrm{H})$ and individuals with $p<2$ per cent (h). 
ranged from 1 to 81 . In each line, and at the three temperatures, the foreign siIII mtDNA frequency increased over generations but it was far more rapid at $25^{\circ}$ than at $21^{\circ}$ or $17^{\circ} \mathrm{C}$. The changes in the level of heteroplasmy in the progeny of one female are shown in Fig. 3; the histograms show the siIII mtDNA distribution at three generations for each temperature. The $\mathrm{F}_{7}$ flies carried only a siIII mitochondrial type at $21^{\circ} \mathrm{C}$ (histogram $\mathrm{f}, 15$ individuals) and $25^{\circ} \mathrm{C}$ (histogram j, 30 individuals). The relative fitness of siIII mtDNA against maII mtDNA, calculated between a mother and its $F_{1}$, is 1.6 at $17^{\circ}$, 2.0 at $21^{\circ}$ and 3.1 at $25^{\circ} \mathrm{C}$.

\section{Discussion}

\section{Nature of the injected material}

Cytoplasm was injected, not purified mitochondria. In general it is consequently inferred that all genetic information transferred was organelle DNA. However, in one case Wolbachia could have been introduced together with siII mitochondria because the presence of these bacteria was detected in the siII strain used. Indeed, it has been demonstrated that Wolbachia can be transmitted in this way (Rousset \& de Stordeur, 1994) and may cause fertility reductions. Because in the present experiments no problem of fecundity was noticed, it was inferred that there was no evidence of competition or co-operation between the organelles and the bacteria.

\section{mtDNA or mitochondria?}

Because the siIII and maI mtDNA are indistinguishable at the RFLP level, the comparison of the $m a \mathrm{I}$ change in frequencies with that of siIII in the presence of maII (Fig. 2b,e) or si (Fig. 2a,c) might have revealed discrete differences. In fact this expectancy was not fulfilled and similarity between these two DNAs is confirmed. This also means that the mitochondrial genome is important when a difference is observed, and that the proteins made by the original nucleus are not, because they were replaced by endogenous proteins after injection. However, comparison of Fig. 1(b) with Fig. 1(c) shows that the changes in siII frequency in the presence of $m a \mathrm{I}$ or siIII are not identical. This may result from (i) the nature of the recipient strains which are very different (bearing nuclei from two different species) and (ii) the foreign mtDNA frequency which was low in the founder females (drift effect).

\section{Not all mtDNAs are equivalent}

In addition to data reported by Solignac et al. (1984) and de Stordeur et al. (1989), the present results indicate that stochastic events are involved in the resolution of heteroplasmy. They are, however, strongly affected by selective pressures because they depend on the nature of the injected mitochondrial type: siII is the most efficient settler $\left(w_{\text {sill }}>1\right.$ in each case, with maybe one exception when injected into $m a \mathrm{I}$ cytoplasm), followed by $m a \mathrm{I}\left(w_{m a \mathrm{I}}>1\right.$, except when injected into siII eggs), siIII ( $w_{\text {sill }}>1$, except when confronted with siII) and finally siI and maII, that were never detected when injected and whose frequency decreased when they were resident confronted with any of the other three mtDNAs.

The selective pressure against $m a \mathrm{II}$ is more important at $25^{\circ}$ than at $21^{\circ}$ or $17^{\circ} \mathrm{C}$ in heteroplas$\mathrm{mic}$ siIII/maII individuals. In artificially heteroplasmic D. melanogaster/D. mauritiana I flies (Matsuura et al., 1991), the frequency of the maI mtDNA (analogous to siIII) increased at $25^{\circ}$ but decreased at $19^{\circ} \mathrm{C}$. In intraspecific heteroplasmic $D$. melanogaster these authors also detected an influence of temperature. Matsuura et al. (1993), using D. melanogaster and $D$. simulans, reported analogous results. Thus the mitochondrial DNA molecule is not neutral and its selective value depends on the relative nature of its environment (both the nuclear genotype and the occurrence of a different mitochondrial genetic information).

\section{Competition levels}

The competition between resident and alien mitochondrial genomes needs to be examined at two levels: intracellular and interindividual.

Intracellular level The injection necessarily causes physical disorders in the recipient eggs; $a$ priori the foreign cytoplasm can be physically rejected or lysed or accepted.

If the two types of mitochondria are present in a cell, they may be in competition and the interaction between nucleus and mitochondria becomes critical. The nucleus of a donor cell could use two general procedures to control a foreign organelle. The first and most likely way bears on mtDNA replication (and thus the number of organelles): its speed could be moderated by the length and by the organization of the mtDNA. The second way could occur if the nuclear-produced proteins fit better in one type of organelle than in the other one.

Another aspect is the ability of the mitochondria to carry on their physiological functions: the results 
of the temperature experiment could be explained by the better ability of siIII mitochondria to work at high temperatures when the metabolic reactions are more rapid and are favoured by effective organelles.

If foreign mitochondria become more and more numerous, it means that the proteins produced by the resident nuclear genome are well adapted to them, possibly better than to the endogenous ones, and/or that the speed of replication of the mitochondria is higher, and/or that these mitochondria are more efficient for cell respiration. The fact that the introduced $m a \mathrm{II}$ or siI mtDNA were never detected in the offspring could be explained by the exclusion of alien mitochondria or by their inability to work in a foreign nuclear context. The siII, siIII and $m a \mathrm{I}$ mtDNAs, accepted by all types of nuclei, might by contrast be described as 'universal'.

The occurrence of intracellular competition between organelles is revealed by a difference between the heteroplasmic state of a founder female and the mean heteroplasmy in its $F_{1}$; the intermitochondrial competition keeps acting in heteroplasmic cells in successive cell generations, particularly when the siII, siIII or maI mtDNA frequency increases over generations.

If Wolbachia are present in a cell, they are competitors for oxygen. Cells harbouring more effective mitochondria could thus be selected when Wolbachia are present. Here, the siII strain was infected and its mtDNA (or mitochondria) advantaged.

The competition occurs also at the intercellular level, between cells harbouring different proportions of both mitochondria, but this level was not examined.

Interindividual level The competition is interindividual when different homoplasmic and/or heteroplasmic individuals are generated in a progeny. In fact, the changes in the mtDNA frequencies between the $F_{1}$ and the last studied generation are roughly the same as between the founder female and its $F_{1}$ (Figs 1-3).

The interindividual and intracellular competition are thus acting in the same direction.

\section{Comparison with introgression by crosses}

Our experiments on mitochondrial introgression through injection, especially when the foreign mitochondrial genome siIII or maI replaces the resident maII (the nuclear resident genome being mauritiana), can be compared to the work of Aubert \& Solignac (1990) where one siIII virgin female was introduced into a bottle containing 32 maII female flies and 33 mauritiana males: after three generations the siIII mtDNA frequency had increased from 3 per cent to 80-100 per cent. In that case the nuclear genome was not pure $D$. mauritiana, but the $D$. simulans nuclear genes were rapidly diluted (the hybrid males being sterile). The introgression was not only mitochondrial and some debate concerned the effect of a residual nuclear introgression. In the present work no nuclei were injected and the results are very similar. We can conclude that, in both situations, the mitochondria (mtDNA) were the determining factor of the invasion. We suppose that the selected mtDNA type conditions a better fertility and/or adaptability to the environment. Maybe the 88 per cent of $m a \mathrm{I}$ mtDNA in $D$. mauritiana natural populations (Solignac \& Monnerot, 1986) reflects an advantage this DNA confers on individuals.

Nigro (1994) interpreted the results of her experiments with competing flies bearing different mtDNAs, in terms of both selection on mtDNA and nuclear-cytoplasmic interaction.

\section{Correlation between selective value and mtDNA geographical distribution}

Taking into account the present geographical distribution of mtDNA, the fact that the siII type is present worldwide could be related to the functional advantage of this mtDNA type. The phylogenetic mitochondrial relationships among these mtDNAs (Solignac \& Monnerot, 1986; Solignac et al., 1986) show that the prevalent types siII and siIII are the closest mtDNAs (nucleotide distance $=1.5$ per cent).

In conclusion, the segregation of mtDNAs in a heteroplasmic individual or in populations bearing two mtDNAs is affected by stochastic processes and by strong selection pressures.

\section{Acknowledgements}

This work was performed in the Centre de Génétique moléculaire, CNRS, Gif-Sur-Yvette, France. We are grateful to Jean-Claude Mounolou, Monique Monnerot and Michel Solignac for continuous interest and for reading an earlier version of this paper.

\section{References}

AUbert, J. AND SOlignaC, M. 1990. Experimental evidence for mitochondrial DNA introgression between Drosophila species. Evolution, 44, 1272-1282.

(C) The Genetical Society of Great Britain, Heredity, 79, 615-623. 
BINNINGTON, K. C. AND HOFFMANN, A. A. 1989. Wolbachialike organisms and cytoplasmic incompatibility in Drosophila simulans. J. Invert. Pathol., 54, 344-352.

CASANE, D., DENNEBOUY, N., DE ROCHAMBEAU, H., MOUNOLOU, J.-C. AND MONNEROT, M. 1994. Genetic analysis of systematic mitochondrial heteroplasmy in rabbits. Genetics, 138, 471-480.

DAWID, I. B. AND BLACKLER, A. W. 1972. Maternal and cytoplasmic inheritance of mitochondrial DNA in Xenopus. Devl. Biol., 29, 152-161.

DE STORDEUR, E., SOlignAC, M., MONNEROT, M. AND MOUNOLOU, J.-C. 1989. The generation of transplasmic Drosophila simulans by cytoplasmic injections: effects of segregation and selection on the perpetuation of mitochondrial DNA heteroplasmy. Mol. Gen. Genet., 220, 127-132.

ENNAFAA, H., MONNEROT, M., EL GAAIED, A. AND MOUNOLOU, J.-C. 1987. Rabbit mitochondrial DNA: preliminary comparison between some domestic and wild animals. Génét. Sél. Évol., 19, 279-288.

GYLLENSTEN, U., WHARTON, D., JOSEFSSON, A. AND WILSON, A. C. 1991. Paternal inheritance of mitochondrial DNA in mice. Nature, 352, 255-257.

HAUSWIRTH, W. W. AND LAIPIS, P. J. 1985. Transmission genetics of mammalian mitochondria: a molecular model and experimental evidence. In: Quagliarello, E., Slater, E. C., Palmieri, F., Saccone, C. and Kroon, A. M. (eds) Achievements and Perspectives of Mitochondrial Research, vol. II, pp. 49-59. Elsevier, New York.

HOEH, W. R., BLAKLEY, K. H. AND BROWN, W. M. 1991. Heteroplasmy suggests limited biparental inheritance of Mytilus mitochondrial DNA. Science, 251, 1488-1490.

KONDO, R., SATTA, Y., MATSUURA, E. T., ISHIWA, H., TAKAHATA, N. AND CHIGUSA, S. I. 1990. Incomplete maternal transmission of mitochondrial DNA in Drosophila. Genetics, 126, 657-663.

LATORRE, A., MOYA, A. AND AYALA, F. J. 1986. Evolution of mitochondrial DNA in Drosophila subobscura. Proc. Natl. Acad. Sci. U.S.A., 83, 8649-8653.

MAGOUlas, A. AND zouros, E. 1993. Restriction-site heteroplasmy in anchovy (Engraulis encrasicolus) indicates incidental biparental inheritance of mitochondrial DNA. Mol. Biol. Evol., 10, 319-325.

MATSUURA, E. T., CHIGUSA, S. I. AND NIKI, Y. 1989. Induction of mitochondrial DNA heteroplasmy by intra- and interspecific transplantation of germ plasm in Drosophila. Genetics, 122, 663-667.

MATSUURA, E. T., NIKI, Y. AND CHIGUSA, S. I. 1991. Selective transmission of mitochondrial DNA in heteroplasmic lines for intra- and interspecific combinations in Drosophila melanogaster. Jap. J. Genet., 66, 197-207.

MATSUURA, E. T., NIKI, Y. AND CHIGUSA, S. I. 1993. Temperature-dependent selection in the transmission of mitochondrial DNA in Drosophila. Jap. J. Genet., 68, 127-135.
MONNEROT, M., MOUNOLOU, J.-C. AND SOlignAC, M. 1984. Intraindividual length heterogeneity of Rana esculenta mitochondrial DNA. Biol. Cell, 52, 811-819.

NIGRO, L. 1994. Nuclear background affects frequency dynamics of mitochondrial DNA variants in Drosophila simulans. Heredity, 72, 582-586.

NIKI, Y., CHIGUSA, s. I. AND MATSUURA, E. T. 1989. Complete replacement of mitochondrial DNA in Drosophila. Nature, 341, 551-552.

RAND, D. M. AND HARRISON, R. G. 1986. Mitochondrial DNA transmission genetics in crickets. Genetics, 114, 955-970.

Rousset, F. AND DE STORdeur, E. 1994. Properties of Drosophila simulans strains experimentally infected by different clones of the bacterium Wolbachia. Heredity, 72, 325-331.

SANTAMARIA, P. 1987. Injecting eggs. In: Roberts, D. B. (ed.) Drosophila: A Practical Approach, pp. 159-173. IRL Press, Oxford.

SATTA, Y., TOYOHARA, N., OHTAKA, C., TATSUNO, Y., WATANABE, T. K., MATSUURA, E. T. ET AL. 1988. Dubious maternal inheritance of mitochondrial DNA in $D$. simulans and evolution of D. mauritiana. Genet. Res., 52, $1-6$.

SKIBINSKI, D. O. F., GALlAGER, C. AND BEYNON, C. M. 1994. Sex-limited mitochondrial DNA transmission in the marine mussel Mytilus edulis. Genetics, 138, 801-809.

SOLIGNAC, M. AND MONNEROT, M. 1986. Race formation, speciation and introgression within the three homosequential species Drosophila simulans, D. mauritiana and D. sechellia inferred from their mitochondrial DNA analysis. Evolution, 40, 531-539.

SOLIGNAC, M., MONNEROT, M. AND MOUNOLOU, J.-C. 1983. Mitochondrial DNA heteroplasmy in Drosophila mauritiana. Proc. Natl. Acad. Sci. U.S.A., 80, 6942-6946.

SOLIGNAC, M., GÉNERMONT, J., MONNEROT, M. AND MOUNOLOU, J.-C. 1984. Genetics of mitochondria in Drosophila mtDNA inheritance in heteroplasmic strains of $D$. mauritiana. Mol. Gen. Genet., 197, 183-188.

SOLIGNAC, M., MONNEROT, M. AND MOUNOLOU, J.-C. 1986. Mitochondrial DNA evolution in the melanogaster species subgroup of Drosophila. J. Mol. Evol., 23, 31-40.

SOLIGNAC, M., GÉNERMONT, J., MONNEROT, M. AND MOUNOLOU, J.-C. 1987. Drosophila mitochondrial genetics: evolution of heteroplasmy through germ line cell divisions. Genetics, 117, 687-696.

VOLZ-LINGENHOHL, A., SOLIGNAC, M. AND SPERLICH, D. 1992. Stable heteroplasmy for a large-scale deletion in the coding region of Drosophila subobscura mitochondrial DNA. Proc. Natl. Acad. Sci. U.S.A., 89, 11525-11532.

zouros, E., FREEMAN, K. R., BALl, A. O. AND POGSON, G. H. 1992. Direct evidence for extensive paternal mitochondrial DNA inheritance in the marine mussel Mytilus. Nature, 359, 412-414. 